\title{
SÍNTESE DA PENEIRA MOLECULAR MCM-41 DERIVADA DA CINZA DA CASCA DO ARROZ
}

R. M.Braga'*, N. M. Teodoro' ${ }^{2}$, F. M. Aquino', J. M. F. Barros ${ }^{3}$, D. M. A. Melo' ${ }^{2}$, J. C. O. Freitas ${ }^{2}$

'Departamento de Eng. de Energias Renováveis-Centro de Energias Alternativas e Renováveis (CEAR)-UFPB.

${ }^{2}$ Laboratório de Tecnologia Ambiental (LABTam)-NUPPRAR-Instituto de Química-UFRN.

${ }^{3}$ Centro de Educação e Saúde-Unidade Acadêmica de Educação, Cuité-UFCG.

*renata@cear.ufpb.br-najarateodoro@hotmail.com - flávia@cear.ufpb.br- joanamfb@gmail.com daraujomelo@gmail.com - juliofreitasj@hotmail.com

\section{RESUMO}

As peneiras moleculares MCM-41 têm atraído grande interesse devido as suas propriedades de alta área específica, volume e diâmetro de poros controláveis. Estes materiais são aplicados em diversos ramos da indústria química como adsorventes, suportes catalíticos e catalisadores heterogêneos em vários processos. Neste trabalho, o MCM-41 foi sintetizado a partir da cinza da casca do arroz, natural e tratada quimicamente, como fontes alternativas de sílica, denominadas CCAMCM-41 e CCAL-MCM-41, respectivamente, com o objetivo de reaproveitar este resíduo e produzir um material mesoporoso de menor custo. Os materiais foram sintetizados pelo método hidrotérmico a partir de um gel com composição molar: CTAMBr $1,0 \mathrm{~mol} \mathrm{~L}^{-1} ; \mathrm{SiO}_{2}$ 4,0 $\mathrm{mol} \mathrm{L}^{-1} ; \mathrm{Na}_{2} \mathrm{O} 1,0 \mathrm{~mol} \mathrm{~L}^{-1} ; \mathrm{H}_{2} \mathrm{O} 200 \mathrm{~mol} \mathrm{~L}^{-1} \mathrm{e}$ caracterizados por FRX, DRX, FT-IR e determinação de área superficial pelo método BET. Os materiais obtidos apresentaram elevada área superfícial específica $(905$ $\mathrm{m}^{2} \mathrm{~g}^{-1}$ ), com dimensões e volume de poros dentro das especificações de materiais mesoporosos, sendo estas prorpriedades semelhantes ao MCM-41 sintetizado com a sílica gel comercial, comprovando que a cinza da casca do arroz pode ser utilizada como fonte alternativa de sílica na síntese de peneiras moleculares do tipo MCM41.

PALAVRAS-CHAVE: MCM-41, síntese hidrotérmica, sílica, cinza da casca do arroz

\section{SYNTHESIS OF MCM-41 MOLECULAR SIEVE DERIVED FROM RICE HUSK ASH}

\section{ABSTRACT}

MCM-41 molecular sieves attracted great interest due their properties, as high specific surface area, controllable diameter and pore volume. These materials have been applied as adsorbents, catalyst supports, and heterogeneous catalysts in various branches of the chemical industry. In this work, MCM-41 was synthesized from rice husk ash, natural and chemically treated, as alternative sources of silica, called CCAMCM-41 and CCAL-MCM-41, respectively, with the aim of reusing this residue and produce a lower cost mesoporous material. The materials were synthesized by the hydrothermal method from a gel composition: CTAMBr 1,0 mol L-1; $\mathrm{SiO}_{2} 4,0 \mathrm{~mol} \mathrm{~L}^{-1} ; \mathrm{Na}_{2} \mathrm{O} 1,0 \mathrm{~mol} \mathrm{~L}^{-1}$; $\mathrm{H}_{2} \mathrm{O} 200 \mathrm{~mol} \mathrm{~L}^{-1}$; and characterized by XRF, XRD, FT-IR and specific surface area determination by the $B E T$ method. The materials showed high specific surface area $\left(905 \mathrm{~m}^{2} \mathrm{~g}^{-1}\right)$, with dimensions and pore volume within the specifications of mesoporous materials, which are proprieties similar to MCM-41 synthesized with commercial silica gel, proving that rice husk ash can be used as an alternative source of silica for the synthesis of MCM-41 molecular sieves.

KEYWORDS: MCM-41, hydrothermal synthesis, silica, rice husk ash. 


\section{SÍNTESE DA PENEIRA MOLECULAR MCM-41 DERIVADA DA CINZA DA CASCA DO ARROZ}

\section{INTRODUÇÃO}

As peneiras moleculares do tipo MCM-41, materiais desenvolvidos pelos pesquisadores da Mobil Oil Corporation, têm atraído grande interesse desde sua descoberta nos anos 90. Suas propriedades físicas como alta área específica, volume e diâmetro de poros controláveis, permitem que sejam aplicadas como adsorventes, suportes catalíticos e catalisadores heterogêneos em diversos ramos da indústria química.

A síntese do MCM-41 é realizada em condições aquosas alcalinas, utilizando basicamente diferentes fontes de sílica e o direcionador da estrutura. $O$ gel preparado, em diferentes razões molares surfactante/silício, é submetido a um tratamento hidrotérmico, em seguida, lavado e seco à temperatura ambiente. A remoção do direcionador pode ocorrer através da extração por solvente líquido, removendo parte dos cátions do direcionador associados com os grupos silanóis. Os cátions restantes serão eliminados pela calcinação, à temperatura aproximada de 550 ${ }^{\circ} \mathrm{C}$ por 3 horas $[1,2]$.

A partir desta típica rotina, as condições de síntese podem ser alteradas com finalidade de obter materiais com diferentes propriedades. Os principais fatores que afetam as propriedades do MCM-41 são a razão molar entre o direcionador e a sílica, a concentração e o comprimento da cadeia carbônica do direcionador, a temperatura de síntese, o $\mathrm{pH}$ e a natureza da sílica $[2,3]$. Quanto à fonte de sílica, normalmente utiliza-se silicato de sódio, tetraetilortosilicato (TEOS), tetrametilamônio silicato (TMA-silicato) e sílica amorfa (Waterglas, Aerosil, Ludox) [4]. Porém, um inconveniente desses precursores de silício é o elevado custo, que resulta em maior custo de produção do material.

Alguns pesquisadores investigam diferentes fontes de sílica para produzir os silicatos mesoporosos, particularmente o MCM-41. A cinza de carvão foi utilizada por Hui e Chao (2006), através do tratamento com uma solução $\mathrm{NaOH} 2 \mathrm{molL}^{-1}$ sob agitação, à $100{ }^{\circ} \mathrm{C}$ durante 4 a 5 horas. O MCM-41 obtido apresentou estrutura hexagonal, porém não organizada, área específica de $900 \mathrm{~m}^{2} \mathrm{~g}^{-1}$ e espessura de parede de $1,0 \mathrm{~nm}$ [5]. O quartzo e o rejeito de diatomita foram utilizados por Silva (2009) como fonte de sílica e alumínio, respectivamente, na síntese de peneiras moleculares mesoporosas do tipo MCM-41 e AIMCM-41. O material resultante apresentou alta área específica e estrutura hexagonal ordenada [4]. Jang [6] sintetizou os materiais mesoporosos MCM-41, MCM-48 e SBA-15 utilizando silicato de sódio extraído a partir da cinza da casca do arroz, que foi submetida tratamento térmico a $700{ }^{\circ} \mathrm{C}$, por 4 horas, seguida de refluxo com solução de $\mathrm{NaOH} 2 \mathrm{molL}^{-1}$ por 24 horas.

A casca do arroz é o subproduto mais volumoso da indústria do arroz. O alto teor de sílica faz desse resíduo uma matéria-prima valiosa que pode ser utilizada na produção do carbeto de silício, como carga em compostos de borracha natural, como materiais pozolânicos em cimentos e concretos, entre outras aplicações em vários setores da indústria.

Alguns materiais zeolíticos tais como ZSM-5 [7,8], ZSM-48 [9], MCM-48 [10], a zeólita beta [11] e mordenita [12] sintetizados a partir de cinzas da casca de arroz foram relatados na literatura. A sua utilização na substituição de sílicas convencionalmente utilizadas em produção de zeólitas, 
suportes catalíticos, produção de silicatos, entre outros produtos, diminui a toxicidade do processo e aumenta a sua economia por se tratar de um material natural e de baixo custo.

Existem muitos métodos de extração de sílica de fontes naturais. Muitos autores têm sugerido que o tratamento ácido antes do tratamento térmico é um método adequado para extração natural da sílica $[5,13,14,15]$. Sílicas de alta pureza e baixo percentual de traços minerais foram recuperadas pela fervura de solução clorídica por 1 hora, seguida de aquecimento à $650-700{ }^{\circ} \mathrm{C}$ por 4 horas [13]. Através deste processo, a sílica extraída possui estrutura amorfa, altamente reativa para ser empregada na síntese de novos materiais.

Este trabalho tem como objetivo sintetizar a peneira molecular mesoporosa do tipo MCM-41 utilizando a cinza da casca do arroz como fonte de sílica, comparando a eficiência da sílica presente na cinza da casca do arroz natural e tratada quimicamente com $\mathrm{HCl}$, com os resultados do MCM-41 sintetizado com sílica gel comercial.

\section{EXPERIMENTAL}

\section{Extração da sílica}

A casca do arroz, originada da região de Caicó - RN, foi lavada com água aquecida a $60{ }^{\circ} \mathrm{C}$, sob agitação constante, por aproximadamente 30 minutos. Este material foi seco, submetido à moagem em moinho de facas (TECNAL TE 631) e calcinado em um forno tipo mufla (TECNAL 3000) a $600{ }^{\circ} \mathrm{C}$, sob taxa de $10{ }^{\circ} \mathrm{C} \mathrm{min}^{-1}$, durante 2 horas. $O$ material resultante, CCA, foi utilizado como fonte de sílica na síntese do CCA MCM-41.

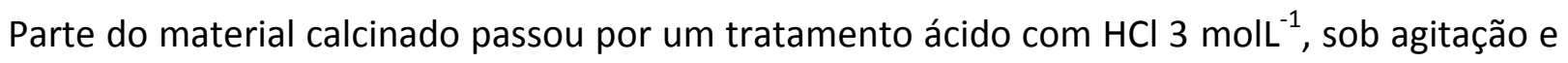
aquecimento, constantes, por 2 horas em ebulição. Em seguida a casca foi lavada com água deionizada até obter $\mathrm{pH}$ entre 5 e 6 . Após seco, o material foi calcinado a $600{ }^{\circ} \mathrm{C}$, sob taxa de 10 ${ }^{\circ} \mathrm{C} \mathrm{min}^{-1}$, durante 2 horas. O material obtido, CCAL, foi utilizado como fonte de sílica para a síntese do material CCAL-MCM-41.

\section{Síntese do MCM-41}

Os materiais foram obtidos através do método hidrotérmico, usando como fontes de sílica: a cinza da casca do arroz calcinada (CCA), cinza da casca do arroz calcinada tratada com $\mathrm{HCl}$ (CCAL) e a sílica gel comercial (MERCK); brometo de cetiltrimetilamônio (CTMABr) como direcionador de estrutura; silicato de sódio e água destilada. O MCM-41 foi sintetizado através da agitação constante de uma solução aquosa contendo a fonte de sílica e o silicato de sódio, a temperatura de $60{ }^{\circ} \mathrm{C}$. Após 2 horas de agitação, uma solução contendo o agente direcionador da estrutura foi adicionada à esta mistura, a temperatura ambiente. $O$ sistema foi mantido sob agitação durante 1 hora. O gel resultante composto por CTAMBr 1,0 mol L-1; SiO $4,0 \mathrm{~mol} \mathrm{~L}^{-1} ; \mathrm{Na}_{2} \mathrm{O}$ 1,0 mol L $\mathrm{m}^{-1} ; \mathrm{H}_{2} \mathrm{O}$ $200 \mathrm{~mol} \mathrm{~L}^{-1}$, foi introduzido em um frasco de teflon com tampa e inserido em um autoclave de aço inox, aquecido em estufa a $100{ }^{\circ} \mathrm{C}$ durante 96 horas. A cada 24 horas foi realizada a correção de $\mathrm{pH}$ ajustado para faixa entre $9-10$, com solução $30 \%$ de ácido acético, até sua estabilização.

A importância do ajuste do $\mathrm{pH}$ se deve ao fato da alcalinidade da mistura reacional favorecer a formação de espécies de silicatos altamente solubilizadas na forma de monômeros e dímeros, sendo essas espécies altamente reativas para o processo de cristalização e formação do MCM-41 [16]. 
O acetato de sódio foi adicionado, para completar a estabilização da sílica, após a estabilização do $\mathrm{pH}$, numa proporção molar direcionador/sal igual a 3, e o sistema foi novamente inserido na estufa por mais 24 horas a $100{ }^{\circ} \mathrm{C}$. O sólido final foi separado do sobrenadante por filtração a vácuo, lavado com solução $2 \%$ em massa de $\mathrm{HCl} /$ Etanol para remoção de parte do sufactante, em seguida com água desionizada, e finalmente seco em estufa a $60^{\circ} \mathrm{C}$. Os materiais sintetizados por esse procedimento foram submetidos a calcinação sob fluxo de $\mathrm{N}_{2}$ de $200 \mathrm{~mL} \mathrm{~min}{ }^{-1}$ a $10^{\circ} \mathrm{C} \mathrm{min}^{-1}$, até $500^{\circ} \mathrm{C}$, permanecendo por 1 hora, em seguida o $\mathrm{N}_{2}$ foi substituído por ar com fluxo de $300 \mathrm{ml}$ $\min ^{-1}$, por mais 1 hora na mesma temperatura.

\section{Caracterização}

A análise química dos materiais precursores de silício foi realizada através de fluorescência de raios $X$ por energia dispersiva (FRX) em um equipamento Shimadzu, modelo EDX-820. Os ensaios de difração de raios $X$ foram realizados em um equipamento da Shimadzu XRD-6000, utilizando a radiação CuKa ( $\lambda=1,5406 \AA$ ) obtida por $30 \mathrm{kV}$ em corrente de filamento de $30 \mathrm{~mA}$. Os dados foram coletados na faixa de $2 \theta$ de 1 a $10^{\circ}$ com velocidade de $2^{\circ} \mathrm{min}^{-1}$ com passo de $0,02^{\circ}$. 0 parâmetro de arranjo hexagonal $\mathrm{a}_{0}$ da estrutura MCM-41 foi obtido através do pico de reflexão do plano (100), o mais intenso no difratograma, sendo calculado pelas Equações 1 e 2:

$1 / d^{2}(100)=4 / 3 a_{0}^{2}$

equação (1)

$\mathbf{a}_{0}=\mathbf{2} \mathbf{d}_{(\mathbf{1 0 0})} / \sqrt{ } \mathbf{3}$

equação (2)

em que d é a distância interplanar relativa ao plano 100, e pode ser calculada pela Equação 3 :

$\lambda_{\mathrm{CuKa}}=2 \mathrm{~d}_{(100)} \operatorname{sen} \theta$

equação (3)

A área superficial específica foi obtida através da adsorção física de $N_{2}$, realizada pelo método Brunauer-Emmett-Teller (BET) em um equipamento da QUANTACHROME NOVA 2000. A espessura da parede de sílica (w) que forma a estrutura hexagonal do MCM-41 foi obtida pela diferença entre o parâmetro de rede $a_{0}$ e o diâmetro do poro (dp), como mostrado na Figura 1.

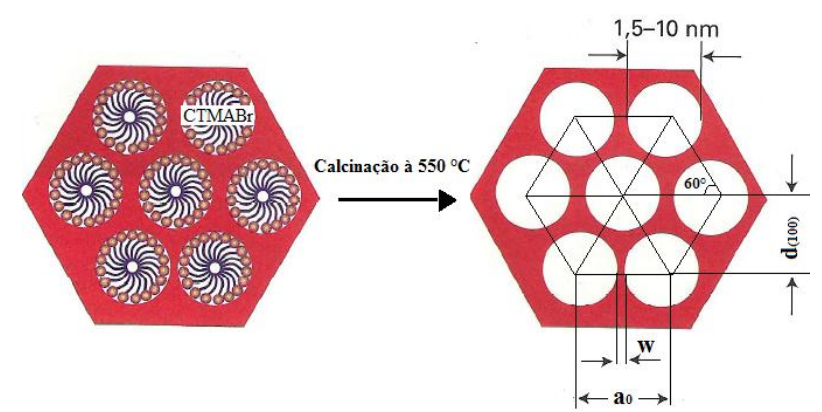

Figura 1 - Representação esquemática do arranjo mesoporoso do MCM-41 antes e após calcinação (Adaptado de Shriver \& Atkins, 2008 [17]). Em que: $w$ - espessura da parede de sílica, $a_{0}$ - parâmetro de arranjo hexagonal e $d_{(100)}$ - distância interplanar relativa ao plano 100.

A análise de espectroscopia na região do infravermelho foi realizada em um espectrofotômetro de infravermelho por Transformada de Fourier da Shimadzu, modelo IRPrestige-21, usando $\mathrm{KBr}$ como agente dispersante. As pastilhas foram preparadas pela mistura de 0,7 mg de amostra com uma quantidade suficiente de $\mathrm{KBr}$ para se atingir a concentração de $1 \%$ em massa. Os espectros foram obtidos na região de 4000 a $400 \mathrm{~cm}^{-1}$. 


\section{RESULTADOS E DISCUSSÃO}

O conhecimento da composição química dos precursores de sílica é fundamental porque o percentual de silício, que compõe cada fonte, influencia nos cálculos estequiométricos dos reagentes necessários para formação do MCM-41. Os resultados de FRX das cinzas da casca do arroz utilizados como fonte de sílica e da sílica comercial, são apresentados na Tabela 1.

Tabela 1 - Composição química das fontes de sílica utilizadas na síntese do MCM-41 através do FRX.

\begin{tabular}{cccc}
\hline $\begin{array}{c}\text { Composição } \\
\text { Química }\end{array}$ & CCA (\%) & CCAL (\%) & SiO $_{2}$ (\%) \\
\hline $\mathrm{SiO}_{2}$ & 95,9 & 98,2 & 99,8 \\
$\mathrm{~K}_{2} \mathrm{O}$ & 2,2 & 0,4 & n.d. \\
$\mathrm{P}_{2} \mathrm{O}_{5}$ & 0,8 & n.d. & n.d. \\
$\mathrm{CaO}$ & 0,5 & 0,1 & 0,08 \\
$\mathrm{MnO}$ & 0,4 & 0,1 & n.d. \\
$\mathrm{Fe}_{2} \mathrm{O}_{3}$ & 0,1 & 0,03 & n.d. \\
Outros & 0,1 & - & 0,05 \\
n.d. = não identificado & & &
\end{tabular}

A composição química da cinza da casca do arroz varia em função do solo em que é plantado, dos tipos e teores dos fertilizantes utilizados, das condições climáticas, bem como do tipo de arroz [13]. Os óxidos presentes na cinza da casca do arroz são minerais que foram absorvidos pelo vegetal ao longo de sua vida. O resultado do FRX mostra que o tratamento ácido foi eficiente na redução do percentual dos óxidos de potássio, fósforo e cálcio presentes na cinza da casca do arroz, resultando em um material com percentual de $\mathrm{SiO}_{2}$ próximo ao da sílica gel comercial (MERCK).

Os difratogramas de raios $X$ das fontes de sílica, Figura 2, apresentaram um pico alargado na varredura de $2 \theta$ igual a $22^{\circ}$, caracterizando comportamento amorfo para esses materiais. Tendo em vista o elevado teor de sílica de estrutura amorfa, estas fontes podem ser consideradas reativas para o processo de síntese do MCM-41.

Os espectros de absorção na região do infravermelho do material precursor de sílica (CCA), bem como do CCA MCM-41 sintetizado, podem ser observados na Figura 3. Na região de 1089 $\mathrm{cm}^{-1}$, para o espectro da CCA, há uma banda intensa, referente ao estiramento assimétrico da ligação Si-O-Si, e em $798 \mathrm{~cm}^{-1}$, observa-se uma banda de menor intensidade, relacionada ao estiramento simétrico desta mesma ligação. A banda que aparece em $3498 \mathrm{~cm}^{-1}$ está relacionada à vibração de deformação axial da hidroxila do grupo Si-OH [18]. Essas bandas também se encontram presentes no CCA MCM-41, comprovando a influência da fonte de sílica na formação da estrutura do material mesoporoso. As bandas que aparecem em 2936 e $2854 \mathrm{~cm}^{-1}$ no espectro do material não calcinado, são atribuídas à deformação axial assimétrica e simétrica, respectivamente, do grupo metileno $\left(-\mathrm{CH}_{2}\right)$ presente no $\mathrm{CTMABr}$, utilizado como direcionador da estrutura do MCM-41. 


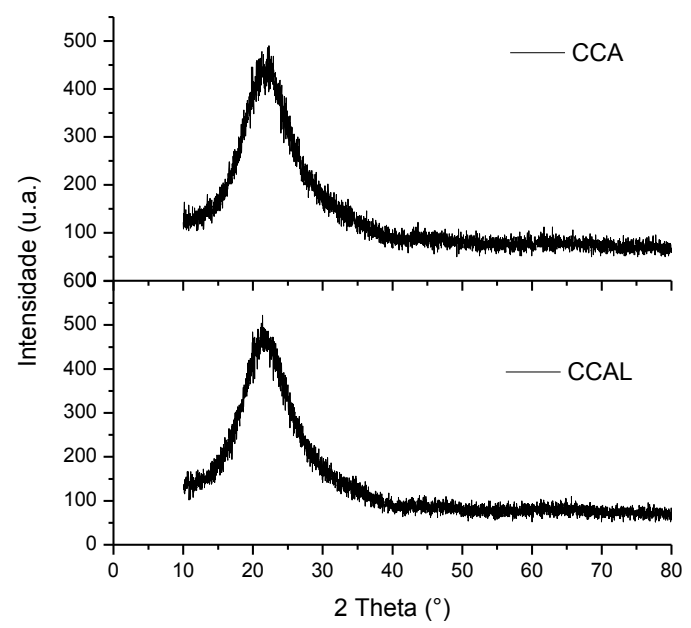

Figura 2 - Difratograma de raios X das fontes de sílica CCA e CCAL.

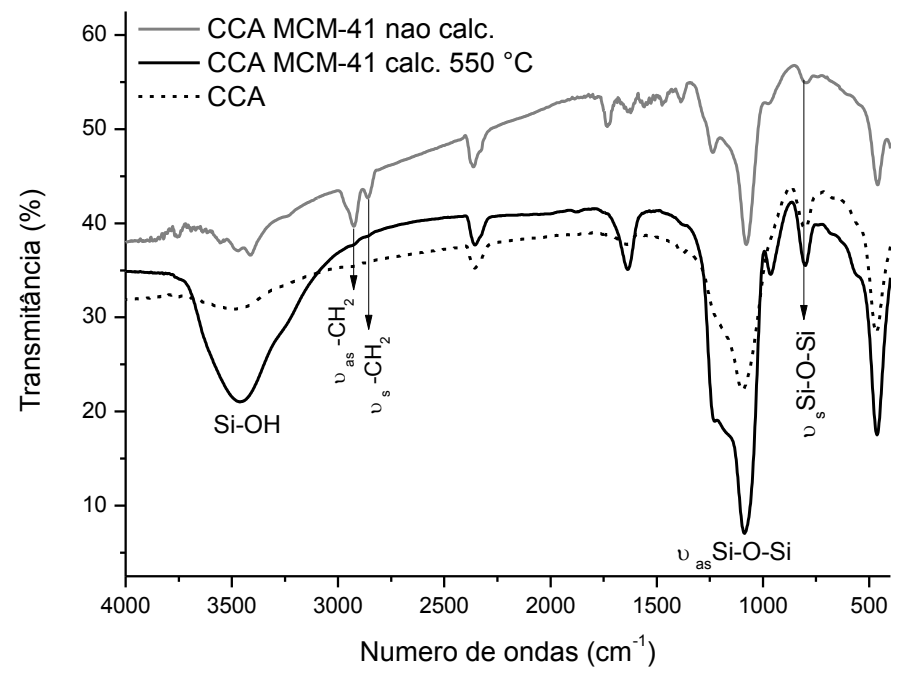

Figura 3 - Espectros de absorção na região do infravermelho da fonte de sílica CCA e do CCA MCM-41 sintetizado.

A Figura 4 apresenta os espectros da fonte de sílica CCAL e dos materiais CCAL-MCM-41, calcinado e não calcinado. A CCAL apresentou bandas mais intensas que a CCA, o que significa a maior abundância das ligações, e consequentemente, maior pureza desse material. Esses resultados podem ser relacionados com a análise de fluorescência de raios $X$, onde o material que foi submetido à lavagem com $\mathrm{HCl}$ apresentou maior percentual de silício, confirmando a eficácia do tratamento ácido na remoção de impurezas. Além das bandas característica das vibrações Si-O-Si, também foi observado às bandas em torno de 2926 e $2856 \mathrm{~cm}^{-1}$, no espectro do material não calcinado, relacionadas às deformações assimétrica e simétrica, respectivamente, do $-\mathrm{CH}_{2}$ presente no $\mathrm{CTMABr}$. Neste mesmo espectro foi identificada uma banda em $1480 \mathrm{~cm}^{-1}$, referente à deformação assimétrica do grupo de cabeça $\left(\mathrm{CH}_{3}-\mathrm{N}^{+}\right)$do CTMABr. Após calcinação, não se observa a presença destas bandas, o que comprova que todo material orgânico foi removido. 


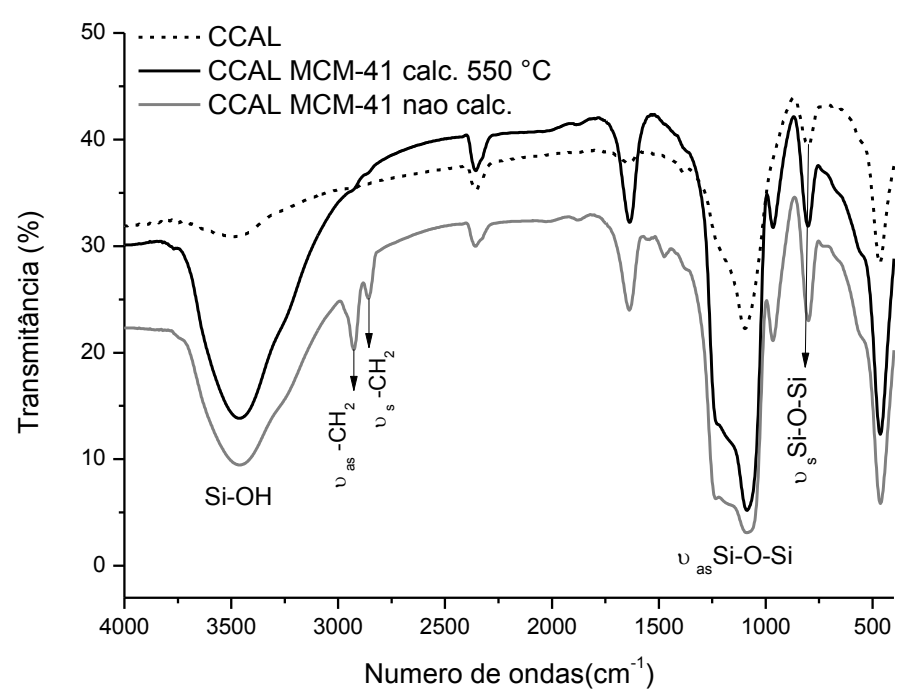

Figura 4 - Espectros de absorção no infravermelho da fonte de sílica CCAL e do CCAL MCM-41 sintetizado.

Os resultados de difração de raios $X$ das amostras CCA MCM-41 e CCAL MCM-41, Figura 5, apresentaram semelhança ao padrão de difratograma do MCM-41, sintetizado com sílica gel comercial. Foram identificados três picos de reflexão em $2 \theta=2,12,3,71$ e 4,27, um com elevada intensidade $(2 \theta=2,12)$, atribuída à linha de reflexão do plano $(100)$, característico da estrutura hexagonal; e dois outros de menores intensidades $(2 \theta=3,71$ e 4,27) atribuídos às reflexões dos planos (110) e (200), que indicam o grau de organização do sistema de poros, como descrito pelos pesquisadores da Mobil Oil Research and Development Co [14].

Os materiais mesoporosos sintetizados possuem boa estabilidade térmica, uma vez que foram calcinados a $550{ }^{\circ} \mathrm{C}$ e ainda apresentaram estrutura com arranjo hexagonal de poros ordenados como mostrado nos difratogramas de raios $\mathrm{X}$ da Figura 5 .

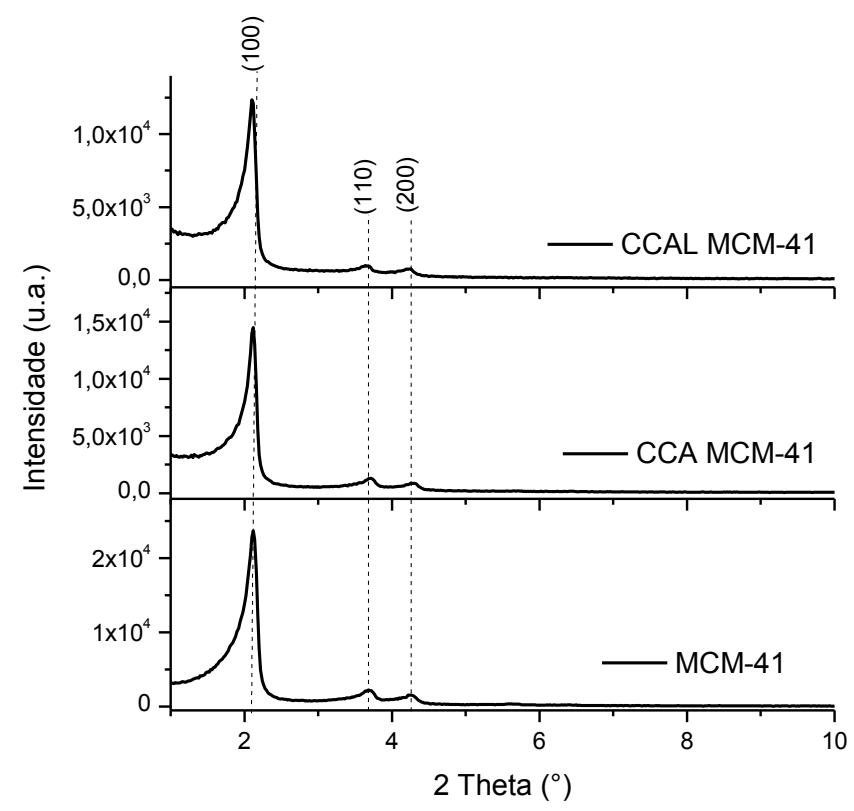

Figura 5 - Difratograma de raios X da CCAL-MCM-41, CCA-MCM-41 e MCM-41 sintetizado com sílica gel. 
Schwanke et al. (2013) sintetizaram a peneira molecular MCM-41 partindo da cinza da casca do arroz, calcinada a $600{ }^{\circ} \mathrm{C}$ durante 4 horas seguida de tratamento químico através de refluxo da solução de $\mathrm{HCl}_{1} \mathrm{molL}^{-1}$, e obtiveram um material que apresentou baixa intensidade dos picos de reflexões dos planos (100), (110) e (200), sugerindo uma estrutura hexagonal desordenada [19].

O pico de reflexão mais intenso, correspondente à reflexão $h k l(100)$ nas amostras dos MCM41 sintetizadas, nos fornece a dimensão do parâmetro de rede hexagonal $\left(\mathrm{a}_{\mathrm{o}}\right)$. Os parâmetros cristalográficos $\mathrm{a}_{0}, \mathrm{~d}_{100}, 2 \theta$ obtidos através da difração de raios $X$ encontram-se na Tabela 2 .

Tabela 2 - Parâmetros cristalográficos dos materiais mesoporosos sintetizados.

\begin{tabular}{cccc}
\hline Amostras & $\mathbf{2 \theta}$ & $\mathbf{a}_{\mathbf{0}}(\mathbf{n m})$ & $\mathbf{d}_{(\mathbf{1 0 0 )}(\mathbf{n m})}$ \\
\hline CCA MCM-41 & 2,12 & 4,81 & 4,17 \\
CCAL MCM-41 & 2,11 & 4,84 & 4,19 \\
MCM-41 & 2,12 & 4,82 & 4,18 \\
\hline
\end{tabular}

Os materiais sintetizados apresentaram elevada área específica, com diâmetro e volume de poros dentro das especificações dos materiais mesoporosos (2 a $10 \mathrm{~nm}$ ) desenvolvidos a partir de fontes convencionais de sílica. A Tabela 3 mostra os resultados das propriedades texturais dos materiais sintetizados. O CCAL MCM-41 apresentou maior área específica, entretanto menor espessura da parede de sílica, quando comparado com o CCA MCM-41. Este resultado sugere uma maior interação elestrostática entre os grupos silanóis e as moléculas do surfactante (CTMABr), podendo ser associado a maior concentração de $\mathrm{SiO}_{2}$ do CCAL, como foi observado nos resultados de FRX e FT-IR.

Tabela 3 - Propriedades texturais dos materiais mesoporosos sintetizados.

\begin{tabular}{cccc}
\hline $\begin{array}{c}\text { Propriedades } \\
\text { texturais }\end{array}$ & CCA MCM-41 & CCAL MCM-41 & MCM-41 \\
\hline Dp $B J H(n m)$ & 3,35 & 3,81 & 3,2 \\
Vp $\left(\mathrm{cm}^{3} \mathrm{~g}^{-1}\right)$ & 0,63 & 0,87 & 0,8 \\
$\mathrm{~S}_{\mathrm{BET}}\left(\mathrm{m}^{2} \mathrm{~g}^{-1}\right)$ & 757 & 905 & 897 \\
$\mathbf{w}(n m)^{*}$ & 1,47 & 1,03 & 1,62 \\
\hline $\mathrm{w}=\mathrm{a}_{0}-\mathrm{dp}$ & & &
\end{tabular}

Os resultados de elevada área específica e ordenamento do arranjo hexagonal de poros comprovam que o simples pré-tratamento da casca do arroz através da lavagem com água aquecida seguida de calcinação a $600{ }^{\circ} \mathrm{C}$ foi satisfatório para obtenção do CCA MCM-41. As metodologias de síntese citadas na literatura requerem diversas etapas para extração da sílica da casca do arroz, e muitas vezes levaram a formação de materiais com propriedades inferiores $[6,19]$.

\section{CONCLUSÕES}

Os resultados apresentados comprovam que a cinza da casca do arroz pode ser utilizada como fonte alternativa de sílica na síntese de peneiras moleculares do tipo MCM-41. Os resultados dos materiais obtidos apresentaram propriedades semelhantes ao do MCM-41 sintetizado com a 
sílica comercial. A fonte de sílica tratada quimicamente apresentou bandas mais intensas na absorção do infravermelho, confirmando a eficiência do tratamento químico na remoção de impurezas. O CCAL MCM-41 apresentou maior área superficial, devido a maior reatividade da sílica após tratamento ácido. Este tratamento é justificado para aplicações que necessite do MCM-41 de alta pureza, pois a cinza da casca do arroz natural atende as exigências para a formação do material mesoporoso. O material obtido pode ser aplicado como adsorvente, catalisador em processos petroquímicos ou suporte catalítico em processos que envolvam temperaturas de até $500{ }^{\circ} \mathrm{C}$, pois acima desta temperatura pode ocorrer a degradação da estrutura hexagonal, característica do $\mathrm{MCM}-41$, e conseqüente alteração das propriedades do material.

\section{REFERÊNCIAS BIBLIOGRÁFICAS}

1. BECK, J.S., VARTULI, J. C., ROTH, W. J., LEONOWICZ, M. E., KRESGE, C. T., SCHMITT, K. D., CHU, C. T. W., OLSON, D. H., SHEPPARD, E. W. A new family of mesoporous molecular sieves prepared with liquid crystal templates. Journal of the American Chemical Society, v. 114, p. 10834-10843, Dec. 1992.

2. SCHUTH, F., BUNSENGES, B. Surface properties and catalytic performance of novel mesostructured oxides. Journal of Physical Chemistry, v. 99, p. 1306, 1995.

3. CHAO, Z-S., RUCKENSTEIN, E. Effect of the nature of the templating surfactant on the synthesis and structure of mesoporous V-Mg-O. Langmuir, v. 18, p. 718-734, Nov. 2002.

4. SILVA, M. L. P. Sínte e caracterização de peneiras moleculares mesoporosas do tipo MCM-41 e Al-MCM-41 a partir de fontes alternativas de sílica e de alumínio. Natal, 2009. Tese de Doutorado - Universidade Federal do Rio Grande do Norte, 2009.

5. HUI, K.S., CHAO, C.Y.H. Synthesis of MCM-41 from coal fly ash by a green approach: Influence of synthesis pH. Journal of Hazardous Materials, v. B137, p. 1135-1148, Apr. 2006.

6. JANG, H.T. et al. Utilization of rice husk ash as silica source for the synthesis of mesoporous silicas and their application to $\mathrm{CO}_{2}$ adsorption through TREN/TEPA grafting. Journal of Hazard Materials, v. 175, p. 928-38, Mar. 2010.

7. CHAREONPANICH, M., NAMTO T., KONGKACHUICHAY P., LIMTRAKUL J. Synthesis of ZSM-5 zeolite from lignite fly ash and rice husk ash. Fuel Process. Technol., v. 85, p. 1623-1634, Oct. 2004.

8. KORDATOS, K., GAVELA, S., NTZIOUNI, A., PISTIOLAS, K.N., KYRITSI, A., KASSELOURIRIGOPOULOU V. Synthesis of highly siliceous ZSM-5 zeolite using silica from rice husk ash. Microporous and Mesoporous Materials, v. 115, p. 189-196, Oct. 2008.

9. WANG, H. P., LIN, K. S., HUANG, Y. J., LI, M. C., TSAUR, L. K. Synthesis of zeolite ZSM-48 from rice husk ash. Journal of Hazardous Materials, v. 58, p. 147-152, 1998.

10. ENDUD, S., WONG, K.L. Mesoporous silica MCM-48 molecular sieve modified with $\mathrm{SnCl}_{2}$ in alkaline medium for selective oxidation of alcohol. Microporous and Mesoporous Materials, v. 101, p. 256-263, 2007. 
11. PRASETYOKO, D. et al. Conversion of rice husk ash to zeolite beta. Waste Management, v. 26, p. 1173-1179, Dec. 2006.

12. BAJPAI, P.K., GOKHALE, K.V.G.K., RAO, M.S. Synthesis of mordenite type zeolite using silica from rice husk ash. Industrial \& Engineering Chemistry Product Research and Development, v. 20, p. 721-726, Dec. 1981.

13. HOTZA, D., DELLA, V.P., JUNKES, J.A., OLIVEIRA, A.P.N. Estudo comparativo entre a sílica obtida por lixívia ácida da casca de arroz e a sílica obtida por tratamento térmico da cinza de casca de arroz. Química Nova, v. 29, p. 1175-1179, Jul. 2006.

14. BECK, J.S. et al. A new family of mesoporous molecular sieves prepared with liquid crystal templates. Journal of the American Chemical Society, v.114, p. 108-34, Dec. 1992.

15. KOH, C. A., NOONEY, R., TAHIR, S. Characterization and catalytic properties of MCM-41 and Pd/MCM-41 materials. Catalysis Letters, v. 47, p. 199, 1997.

16. ALVES, J. A. B. L. R. Síntese e caracterização de MCM-41 contendo terras raras na dessulfurização utilizando tiofeno como molécula sonda. Natal, 2010. Dissertação de Mestrado - Universidade Federal do Rio Grande do Norte, 2010.

17. SHRIVER, D. F., ATKINS, P. Química Inorgânica. 4Ed., Porto Alegre, Bookman, 2008.

18. SILVERSTEIN, R. M. Identificação espectrométrica de compostos orgânicos. 7Ed, 2006.

19. SCHWANKE, A. J., MELO, D. M. A., SILVA, A. O., PERGHER, S. B. C. Use of rice husk ash as only source of silica in the formation of mesoporous materials. Cerâmica, v. 59, p. 181-185, 2013. 